\title{
LEPTIN INCREASES THROMBOXANE A2 FORMATION IN THE RAT
}

\author{
Andrzej Marciniak, Grażyna Wójcicka, Jerzy Bełtowski \\ Department of Pathophysiology, Medical University, Lublin, Poland
}

\begin{abstract}
Chronic hyperleptinemia may contribute to various complications of obesity including atherosclerosis, however, the underlying mechanisms are incompletely clear. We examined the effect of leptin on platelet activity by measuring stable metabolites of thromboxane $A_{2}\left(T X A_{2}\right), T X B_{2^{\prime}}$ 11-dehydro-TXB and 2,3-dinor-TXB ${ }_{2}$ in plasma and urine. In vitro, leptin stimulated $\mathrm{TXB}_{2}$ formation by platelet-rich plasma (PRP). In vivo, leptin (1 mg/kg ip.) increased urinary excretion of 11-dehydro-TXB ${ }_{2}$ and 2,3-dinor$\mathrm{TXB}_{2}$. Urinary excretion of these metabolites was also elevated in rats made hyperleptinemic by administration of recombinant leptin $(0.5 \mathrm{mg} / \mathrm{kg} /$ day $)$ for 8 days. The stimulatory effect of leptin on $\mathrm{TXB}_{2}$ formation in PRP isolated from hyperleptinemic animals was impaired in comparison to the control group. In rats made obese, hyperleptinemic and hyperinsulinemic/insulin resistant by cafeteria diet administered for 3 months, acute stimulatory effect of leptin on $\mathrm{TXB}_{2}$ formation by PRP was not impaired. In rats made insulin resistant by fructose feeding for 8 weeks, stimulatory effect of leptin on $\mathrm{TXB}_{2}$ formation in PRP was augmented in comparison to the control group. Insulin sensitizer, rosiglitazone, decreased insulin level and attenuated the stimulatory effect of leptin on $\mathrm{TXB}_{2}$ formation in obese and fructose-fed animals. In contrast, rosiglitazone had no effect on insulin level or leptin-induced $\mathrm{TXB}_{2}$ formation in control rats and rats receiving recombinant leptin for 8 days. These results indicate that: (i) leptin stimulates platelet TXA $\mathrm{A}_{2}$ formation both in vitro and in vivo, (ii) chronic hyperleptinemia impairs acute stimulatory effect of leptin on platelet activity if insulin sensitivity is normal, (iii) insulin resistance/hyperinsulinemia augments the stimulatory effect of leptin on TXA formation, which results in normal platelet sensitivity to leptin in obesity associated with both hyperleptinemia and hyperinsulinemia, and (iv) PPAR- $\gamma$ agonists such as rosiglitazone decrease platelet sensitivity to leptin by reducing insulin resistance.
\end{abstract}

Adipobiology 2009; 1: 77-85

Key words: platelets, obesity, metabolic syndrome, atherosclerosis, rosiglitazone

Received 30 July 2009, accepted 25 August 2009.

Correspondence and reprint request: Dr Jerzy Bełtowski, Department of Pathophysiology, Medical University, ul. Jaczewskiego 8 , PL-20-090 Lublin, Poland. Tel: +48 81 7187365, Fax: +48 817187364, E-mail: jerzy.beltowski@am.lublin.pl

\section{Introduction}

Recent studies indicate that an adipose tissue hormone, leptin, is involved in atherogenesis. Plasma leptin concentration is markedly increased in obese humans and in animals with obesity induced by high-calorie diet. In experimental studies leptin has been demonstrated to have many potentially proatherogenic effects. This adipokine induces endothelial dysfunction, oxidative stress, vascular smooth muscle cells hypertrophy and proliferation, stimulates macrophage cholesterol synthesis, and reduces HDL cholesterol level (1). Deficiency of leptin or its receptor reduce atherosclerosis in classic animal models such as apolipoprotein $\mathrm{E}$ and LDL receptor knockout mice, whereas administration of exogenous leptin in supraphysiological doses or transgenic hormone overexpression have the opposite effect (2). In addition, many clinical studies indicate the link between high leptin level and atherosclerosis, acute cardiovascular events and ischemic stroke in humans (3).

Platelets play a crucial role in atherosclerosis. Formation of plateletrich thrombus on the ruptured plaque, with resulting complete or almost complete vessel occlusion, is the ma- 
jor mechanism of acute complications of atherosclerosis such as myocardial infarction and ischemic cerebral stroke. In addition, chronic low-grade platelet activation facilitates growth of atherosclerotic plaque. Platelets secrete several mediators which potently stimulate hypertrophy and proliferation of vascular smooth muscle cells including platelet-derived growth factor and thromboxane $\mathrm{A}_{2}\left(\mathrm{TXA}_{2}\right)$. Moreover, activated platelets facilitate leukocyte recruitment to the endothelium by secreting chemotactic and proinflammatory mediators such as a chemokine platelet factor-4, a CD40 ligand, P-selectin, thrombospondin-1 and RANTES (4).

The effect of leptin on platelet function is controversial. Some studies have demonstrated that leptin augments ADP-induced platelet aggregation (5), but other authors observed no effect (6). These studies were performed in vitro, and it is well-known that platelet aggregation in vitro is at best only a crude estimate of their function in the intact organism (7). Although the positive correlation between serum leptin and urinary excretion of TXA $_{2}$ metabolite, 11-dehydro- $\mathrm{TXB}_{2}$, has been observed in obese women (8), this correlation disappeared after adjustment for anthropometric variables suggesting that it represents the effect of obesity and not necessarily of leptin itself. In addition, resistance to many effects of leptin has been described in obesity. It is unclear if platelets' sensitivity to leptin is preserved or impaired in obesity. If platelets remain sensitive to leptin, chronic hyperleptinemia could contribute to platelets' hyperactivity observed in the metabolic syndrome. However, if platelets become resistant to leptin, the contribution of this adipokine to atherothrombotic complications would be less likely.

To address these issues, in the present study we examined the effect of leptin on platelet function, measured as TXA 2 production, in the rat both in vitro and in vivo. In addition, we compared the effect of leptin in lean animals and in selected models of the metabolic syndrome. We also tested the effect of rosiglitazone, a PPAR- $\gamma$ agonist, on leptin-induced TXA2 formation.

\section{Materials and methods}

\section{Animals}

All studies were performed on adult male Wistar rats weighing $314 \pm 8 \mathrm{~g}$. The animals were housed under controlled conditions of temperature $\left(20-22^{\circ} \mathrm{C}\right)$, humidity $(60-70 \%)$, lighting $(12 \mathrm{~h}$ light/dark cycle) and provided with food and water ad libitum. The study protocol was reviewed and approved by the local institutional ethical committee.

\section{Effect of leptin on TXA, production in vitro}

Rats were anesthetized with pentobarbital (50 mg/kg ip.) and blood was withdrawn from the abdominal aorta using sodium citrate as an anticoagulant. Blood was centrifuged at $150 \times \mathrm{g}$ for $15 \mathrm{~min}$ at a room temperature to obtain platelet-rich plasma (PRP). PRP was diluted with Tyrode's buffer (5 mM HEPES, 2 $\mathrm{mM} \mathrm{MgCl}, 0.1 \% \mathrm{BSA}$ and $0.1 \% \mathrm{D}$-glucose) to a standard concentration of $3 \times 10^{8}$ platelets $/ \mathrm{ml}$. To obtain platelet-poor plasma (PPP), PRP was recentrifuged at $1000 \times \mathrm{g}$ for $15 \mathrm{~min}$ and the supernatant was collected. PRP was incubated with leptin for 10 min and $\mathrm{TXB}_{2}$ concentration was measured (see below).

\section{Effect of leptin on TXA, production in vivo}

Rats were anesthetized with ethyluretane ( $1.25 \mathrm{~g} / \mathrm{kg}$ ip.). Then, a polyethylene catheter was inserted into the urinary bladder for urine collection. Urine was collected for 1 hour and then either leptin $(1 \mathrm{mg} / \mathrm{kg}$ in $0.5 \mathrm{ml})$ or an equivolume amount of phosphate-buffered saline (PBS) was injected intraperitoneally. Urine collection was continued for additional 2 hours. After the end of urine collection, animals were euthanized by the lethal dose of pentobarbital.

\section{Induction of hyperleptinemia, dietary-induced obesity and fructose-induced metabolic syndrome}

Experimental hyperleptinemia was induced by administration of exogenous recombinant leptin $(0.25 \mathrm{mg} / \mathrm{kg}$ twice daily sc.) for 8 days as described by us previously (9). Leptin was injected between 7.00 and $8.00 \mathrm{AM}$ and between 7.00 and $8.00 \mathrm{PM}$. The last dose was given in the morning and plasma for in vitro experiments was obtained 6 hours after this injection. Animals in this group received standard rat chow ad libitum. Obesity was induced by offering the animals a highly palatable "cafeteria diet" for either 1 or 3 months. This diet consisted of standard chow combined 1:1 (wt/wt) with a liquid diet containing equal amounts of sucrose, glucose, whole milk powder and soybean powder suspended in tap water (10). The composition of this diet was similar to standard chow (66\% carbohydrates, $20 \%$ protein, and $14 \%$ fat). In the separate group of animals, fructose was administered in the drinking water at a concentration of $20 \%$ for 8 weeks to induce hyperlipidemia and insulin resistance not associated with obesity. In subgroups of control, hyperleptinemic, 3-month obese and fructose-fed animals, insulin sensitizer, rosiglitazone, was administered at $10 \mathrm{mg} / \mathrm{kg} /$ day by oral gavage for 8 days before blood collection for acute in vitro experiments.

Measurement of TXA metabolites

$\mathrm{TXA}_{2}$ is rapidly converted non-enzymatically to its immediate 
metabolite, $\mathrm{TXB}_{2}$, which is further enzymatically metabolized to several derivatives such as 2,3-dinor- $\mathrm{TXB}_{1}, 2,3$-dinor- $\mathrm{TXB}_{2}$ and 11-dehydro- $\mathrm{TXB}_{2}$ (11). Measurement of these terminal metabolites in urine provides a reliable estimate of whole-body plateletderived $\mathrm{TXA}_{2}$. In contrast, because $\mathrm{TXB}_{2}$ has a short half-life (5-7 $\mathrm{min})$, most of this compound found in urine originates from local intrarenal production rather than from systemic sources. On the other hand, concentrations of 2,3-dinor- $\mathrm{TXB}_{2}$ and 11-dehydro- $\mathrm{TXB}_{2}$ in plasma are below detection limit of most assays until the sample is concentrated. However, most of $\mathrm{TXB}_{2}$ found in plasma is synthesized by platelets ex vivo after blood withdrawal. Therefore, we used plasma $\mathrm{TXB}_{2}$ and urinary 11-dehydro- $\mathrm{TXB}_{2} / 2,3$-dinor- $\mathrm{TXB}_{2}$ as markers of $\mathrm{TXA}_{2}$ formation for in vitro and in vivo studies, respectively. $\mathrm{TXB}_{2}, 11$-dehydro- $\mathrm{TXB}_{2}$ and 2,3-dinor- $\mathrm{TXB}_{2}$ were measured by competitive enzyme immunoassay (EIA) methods using Cayman Chemical kits (Cat. \#519031, 519501 and 519051, respectively), according to the manufacturer's instruction. The detection limits are $11 \mathrm{pg} /$ $\mathrm{ml}$ for $\mathrm{TXB}_{2}, 16 \mathrm{pg} / \mathrm{ml}$ for 11-dehydro-TXB ${ }_{2}$, and $7 \mathrm{pg} / \mathrm{ml}$ for 2,3-dinor- $\mathrm{TXB}_{2}$.

\section{Other assays}

Plasma insulin and leptin concentrations were measured by EIA methods using Rat Insulin EIA Kit (SPIbio, Massy, France) and Leptin Enzyme Immunoassay Kit (Cayman Chemical), respectively (10). Lipid profile and plasma glucose were assayed by routine laboratory methods.

\section{Reagents}

Recombinant rat leptin was obtained from R\&D Systems. Rosiglitazone was purchased from Cayman Chemical. Other reagents were from Sigma-Aldrich.

\section{Statistical analysis}

Baseline (without leptin) and leptin-stimulated values of TXA metabolites in plasma and urine of the same animal were compared by repeated-measures ANOVA. Between-group comparisons of TXA 2 metabolites, plasma lipids, glucose, leptin and insulin were done by single-measures ANOVA. $\mathrm{P}<0.05$ was considered significant.

\section{Results}

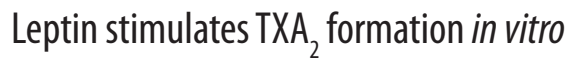

Leptin increased $\mathrm{TXB}_{2}$ concentration in PRP isolated from control lean rats in a concentration-dependent manner (Fig. 1). Leptin had no significant effect at physiological concentration $(10 \mathrm{ng} / \mathrm{ml})$ as well as at a moderately elevated concentration (50 $\mathrm{ng} / \mathrm{ml}$ ), but significantly increased $\mathrm{TXB}_{2}$ at $100 \mathrm{ng} / \mathrm{ml}$. Maximal stimulatory effect of leptin was observed at $300 \mathrm{ng} / \mathrm{ml}$ (Fig. 1). Addition of COX inhibitor, indomethacin $(10 \mu \mathrm{M})$ to the blood before isolation of PRP decreased baseline $\mathrm{TXB}_{2}$ concentration to $243 \pm 29 \mathrm{pg} / \mathrm{ml}$ ( $\mathrm{p}<0.001$ vs. sample without indomethacin) and abolished the stimulatory effect of leptin $(100 \mathrm{ng} / \mathrm{ml}$ leptin + indomethacin: $279 \pm 31 \mathrm{pg} / \mathrm{ml}$ ). In addition, leptin had no significant effect on $\mathrm{TXB}_{2}$ concentration in platelet-poor plasma (without leptin: $479 \pm 57 \mathrm{pg} / \mathrm{ml}$; with $100 \mathrm{ng} / \mathrm{ml}$ leptin: $512 \pm 53$ $\mathrm{pg} / \mathrm{ml}, \mathrm{p}=\mathrm{NS})$.

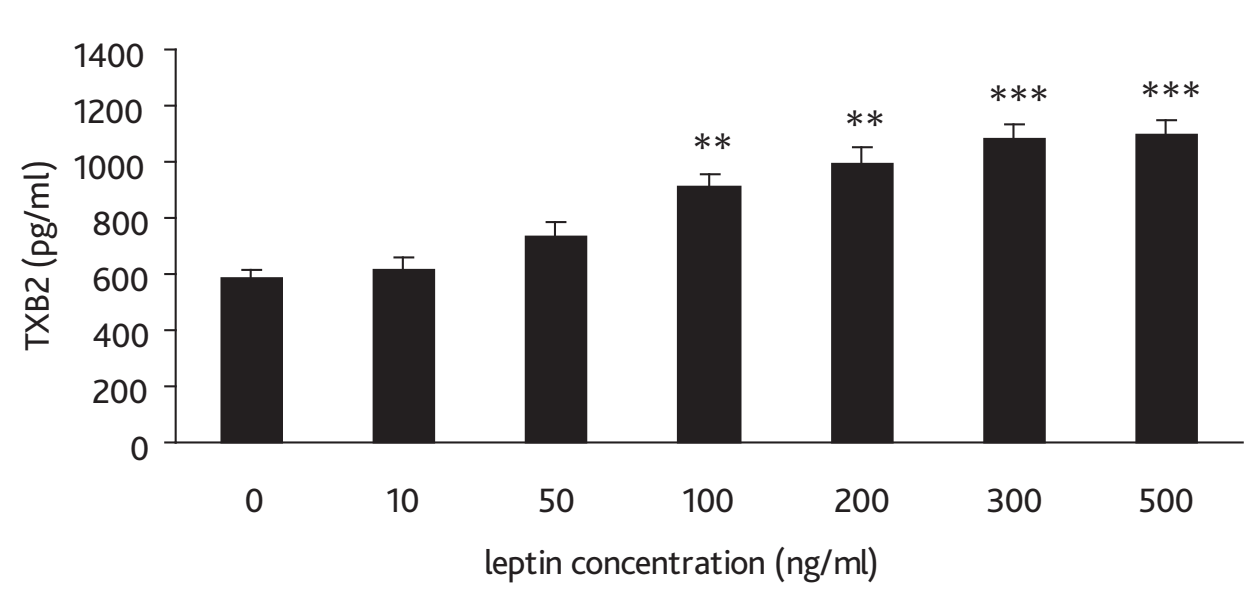

Figure 1. Effect of leptin on thromboxane $B_{2}$ concentration in platelet-rich plasma (PRP) of control rats in vitro. PRP was incubated in the presence of various leptin concentrations for $10 \mathrm{~min}$ and then $T X B_{2}$ was measured. ${ }^{* *} \mathrm{p}<0.01,{ }^{* * *} \mathrm{p}<0.001$ vs. TXB concentration in PRP not treated with leptin. 


\section{Leptin stimulates TXA formation in vivo}

Urinary excretion of 11-dehydro- $\mathrm{TXB}_{2}$ and 2,3-dinor- $\mathrm{TXB}_{2}$ increased within 2 hours after a single intraperitoneal leptin injection (Fig. 2). In contrast, leptin had no acute effect on urinary $\mathrm{TXB}_{2}$. Injection of vehicle (PBS) instead of leptin did not change any TXA ${ }_{2}$ metabolite in urine (not shown).

\section{TXA formation is increased in rats receiving leptin for 8 days}

Plasma $\mathrm{TXB}_{2}$ concentration in rats receiving exogenous leptin for 8 days was $612 \pm 57 \mathrm{pg} / \mathrm{ml}$ and did not differ from control group. However, urinary excretion of 11-dehydro- $\mathrm{TXB}_{2}, 2,3$-dinor- $\mathrm{TXB}_{2}$, and $\mathrm{TXB}_{2}$ was higher in hyperleptinemic than in control animals (Fig. 3). These results indicate that chronic hyperleptinemia increases systemic and intrarenal TXA $\mathrm{T}_{2}$ formation.

\section{Acute effect of leptin on TXA, formation} is impaired in hyperleptinemic rats

To examine if chronic hyperleptinemia induces resistance of platelets to leptin, we obtained PRP from rats made hyperleptinemic by previous 8-day leptin administration and then studied acute effect of leptin on $\mathrm{TXB}_{2}$ formation in these samples in vitro (Fig. 4). As can be seen, in hyperleptinemic rats leptin significantly stimulated $\mathrm{TXB}_{2}$ formation at a concentration no less than $300 \mathrm{ng} / \mathrm{ml}$ and did it to a lesser extent than in control animals at both $300 \mathrm{ng} / \mathrm{ml}$ and $500 \mathrm{ng} / \mathrm{ml}$. Thus, acute stimulatory effect of leptin on platelet $\mathrm{TXA}_{2}$ formation is impaired in hyperleptinemic rats.

\section{Effect of leptin on TXA formation in obese and fructose-fed rats} To examine if chronic "endogenous" hyperleptinemia associated with obesity impairs acute effect of leptin on $\mathrm{TXA}_{2}$ formation, we investigated the effect of leptin on $\mathrm{TXB}_{2}$ concentration in PRP of rats made obese by high-calorie diet administered for either 1 or 3 months (Fig. 5). Baseline (without exogenous leptin) $\mathrm{TXB}_{2}$ concentration was slightly but significantly higher in the 3-month but not in the 1-month obese group. After 10-min

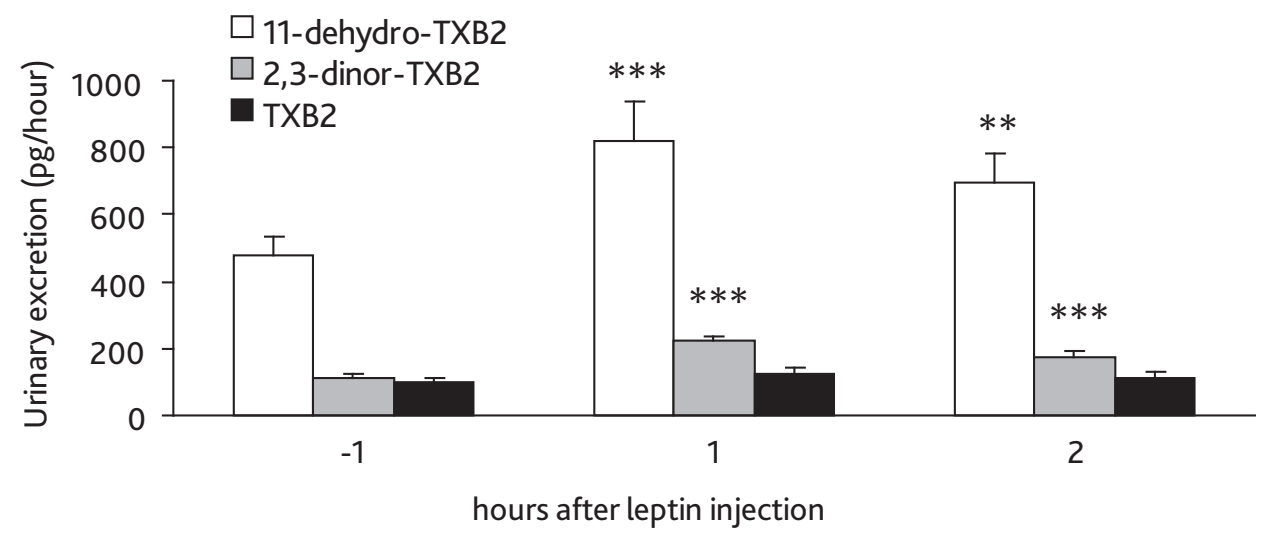

Figure 2. Urinary excretion of TXA metabolites in lean control rats before (-1) and during the first (1) and the second (2) hour after intraperitoneal leptin injection at a dose of $1 \mathrm{mg} / \mathrm{kg} .{ }^{* *} \mathrm{p}<0.01,{ }^{* * *} \mathrm{p}<0.001 \mathrm{vs}$. pre-injection values.

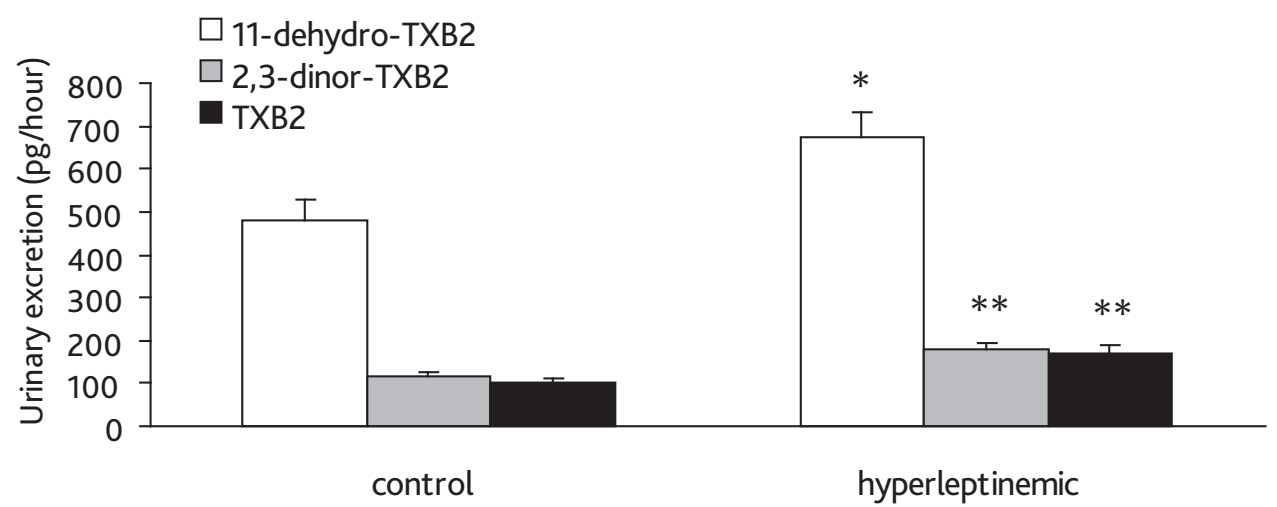

Figure 3. Urinary excretion of TXA $\mathrm{A}_{2}$ metabolites in control rats and in animals receiving exogenous leptin ( $0.25 \mathrm{mg} / \mathrm{kg} \mathrm{sc}$.) for 8 days. Urine was collected for 1 hour in anesthetized animals. Urine collection was started 6 hours after the last leptin injection. ${ }^{*} p<0.05,{ }^{* *} p<0.01$ vs. control group. 


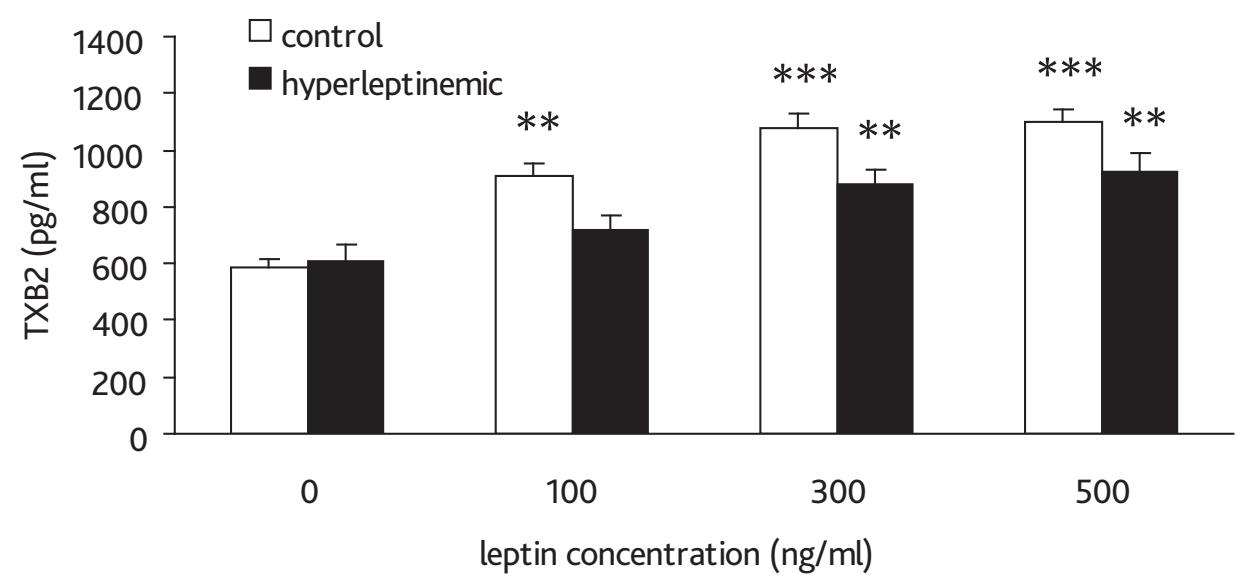

Figure 4. Concentration-dependent effect of leptin on $\mathrm{TXB}_{2}$ in PRP obtained from control rats and from rats made hyperleptinemic by previous 8-days leptin administration. ${ }^{* *} \mathrm{p}<0.01,{ }^{* * *} \mathrm{p}<0.001 \mathrm{vs}$. TXB ${ }_{2}$ concentration in PRP not treated with leptin.

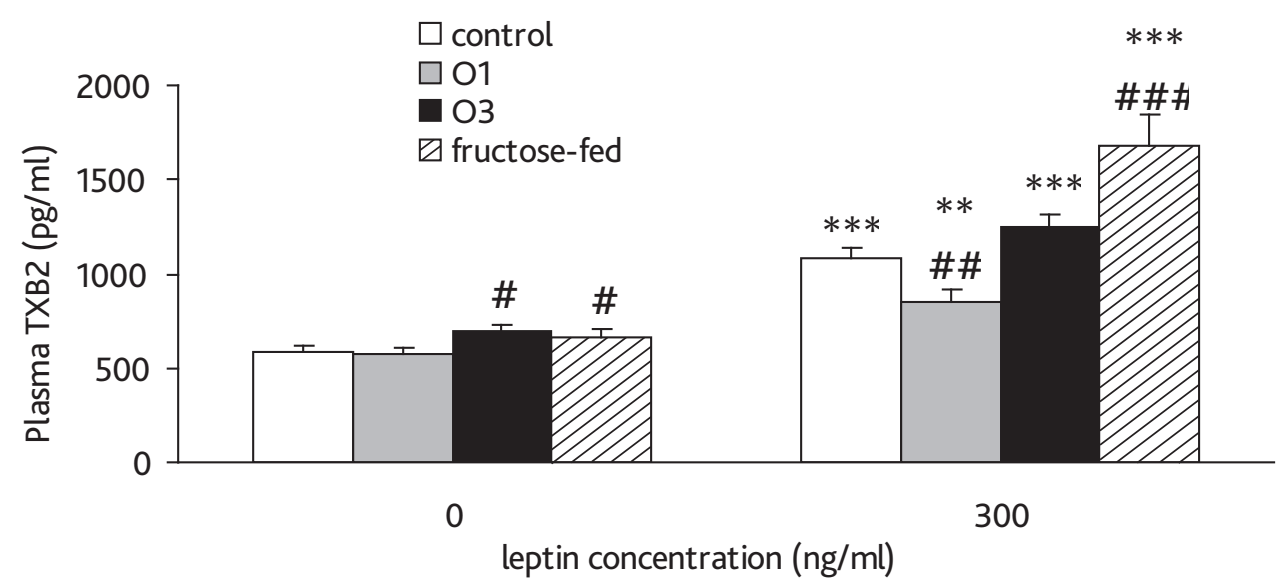

Figure 5. Effect of leptin on $\mathrm{TXB}_{2}$ formation in PRP of control, 1-month obese (O1), 3-month obese (O3) and fructose-fed rats. ${ }^{* *} p<0.01,{ }^{* * *} p<0.001$ vs. $\mathrm{TXB}_{2}$ concentration in the respective sample not treated with leptin, ${ }^{*} p<0.05,{ }^{* \#} p<0.01,{ }^{* \# \#} p<0.001$ vs. respective (treated or not treated with leptin) control group.

incubation, leptin $(300 \mathrm{ng} / \mathrm{ml})$ increased $\mathrm{TXB}_{2}$ concentration in PRP of control animals by $84.1 \%$ but in 1 -month obese group by only $49.6 \%$. Surprisingly, in contrast to short-time obesity, the effect of leptin in 3-month obese group was not impaired in comparison to the control group (stimulation by $80.9 \%$ ).

Metabolic characteristics of both obese groups is presented in Table 1 . As can be seen, plasma leptin is similarly elevated in both obese groups but in contrast to 1-month group, the 3-month group exhibits hyperinsulinemia (a marker of insulin resistance) and dyslipidemia (hypertriglyceridemia and low HDL-cholesterol). Insulin inhibits platelet function and its effect is impaired in insulin resistance states (12). Therefore, we hypothesized that insulin resistance in the 3-month obese group might paradoxically increase the sensitivity of platelets to leptin in comparison to 1-month obese group. To verify this hypothesis, we examined the effect of leptin in fructose-fed rats, which are markedly hyperinsulinemic and hypertriglyceridemic but only slightly hyperleptinemic (Table 1$)$. Leptin $(300 \mathrm{ng} / \mathrm{ml})$ increased $\mathrm{TXB}_{2}$ concentration in PRP of fructose-fed animals to a much greater degree $(+153.0 \%)$ than in control rats. Thus, fructose-induced metabolic syndrome is associated with enhanced platelet response to leptin. Taken together, these results suggest that in the 3-month obese group resistance of platelets to leptin due to chronic hyperleptinemia is counterbalanced by concomitant hyperinsulinemia/insulin resistance which increases platelets' sensitivity to this adipokine. 
Table 1. Characteristics of experimental groups

\begin{tabular}{|l|c|c|c|c|c|}
\hline & Control & Hyperleptinemic & 1-month obesity & 3-month obesity & Fructose-fed \\
\hline Body weight (g) & $357 \pm 5$ & $342 \pm 7$ & $429 \pm 7^{* * *}$ & $497 \pm 6^{* * *}$ & $385 \pm 9^{*}$ \\
\hline Triglycerides (mmol/l) & $0.83 \pm 0.06$ & $0.74 \pm 0.06$ & $0.85 \pm 0.05$ & $1.20 \pm 0.08^{* * *}$ & $2.71 \pm 0.19^{* * *}$ \\
\hline Total cholesterol (mmol/l) & $2.05 \pm 0.21$ & $1.97 \pm 0.17$ & $1.83 \pm 0.11$ & $1.47 \pm 0.12^{*}$ & $2.06 \pm 0.15$ \\
\hline HDL-cholesterol (mmol/l) & $1.24 \pm 0.07$ & $1.25 \pm 0.10$ & $1.19 \pm 0.08$ & $0.76 \pm 0.08^{* * *}$ & $1.30 \pm 0.12$ \\
\hline Plasma glucose (mmol/l) & $6.01 \pm 0.29$ & $6.09 \pm 0.35$ & $6.34 \pm 0.43$ & $6.30 \pm 0.41$ & $6.178 \pm 0.39$ \\
\hline Plasma insulin (ng/ml) & $2.38 \pm 0.24$ & $2.15 \pm 0.20$ & $2.58 \pm 0.32$ & $3.89 \pm 0.46^{*}$ & $4.99 \pm 0.61^{* * *}$ \\
\hline Plasma leptin (ng/ml) & $4.22 \pm 0.41$ & $13.6 \pm 1.23^{* * *}$ & $9.39 \pm 0.81^{* * *}$ & $12.60 \pm 1.27^{* * *}$ & $5.97 \pm 0.52^{*}$ \\
\hline
\end{tabular}

${ }^{*} p<0.05,{ }^{* *} p<0.01,{ }^{* * *} p<0.001$, compared to control group.

\section{Rosiglitazone attenuates the effect of leptin on TXA formation} in the 3-month obese and fructose-fed rats

To further evaluate the above mentioned hypothesis, we examined the effect of peroxisome proliferator-activated receptor- $\gamma$ (PPAR- $\gamma$ ) agonist, rosiglitazone (RGZ), which increases insulin sensitivity, on platelet response to leptin in various experimental groups. Rosiglitazone had no effect on plasma leptin, insulin and lipid profile in either control or hyperleptinemic rats. In addition, rosiglitazone did not alter leptin-induced increase in $\mathrm{TXB}_{2}$ in PRP of these animals (not shown). In the 3-month obese group, rosiglitazone had no significant effect on plasma leptin (without RGZ: $12.60 \pm 1.27 \mathrm{ng} / \mathrm{ml}$; with RGZ: $10.21 \pm 0.98$ $\mathrm{ng} / \mathrm{ml}$ ), but decreased plasma insulin (from $3.89 \pm 0.46$ to 2.57 $\pm 0.29 \mathrm{ng} / \mathrm{ml}, \mathrm{p}<0.01$ ) and triglycerides (from $1.20 \pm 0.08$ to 0.74 $\pm 0.05 \mathrm{mM}, \mathrm{p}<0.01$ ). Baseline (without exogenous leptin) $\mathrm{TXB}_{2}$ concentration was lower in RGZ-treated than in non-treated 3-month obese rats ( $571 \pm 27$ vs. $693 \pm 39 \mathrm{pg} / \mathrm{ml}, \mathrm{p}<0.05)$. In addition, leptin $(300 \mathrm{mg} / \mathrm{ml})$ increased $\mathrm{TXB}_{2}$ concentration in RGZ-treated obese rats to $878 \pm 87 \mathrm{pg} / \mathrm{ml}(+53.8 \%)$, which is significantly less than in either control or RGZ-untreated obese animals. In the fructose-fed group, RGZ decreased plasma insulin $(-54.6 \%)$ and triglycerides $(-41.7 \%)$ and reduced the stimulatory effect of $300 \mathrm{ng} / \mathrm{ml}$ leptin on $\mathrm{TXB}_{2}$ from $+153.0 \%(\mathrm{p}<0.01$ vs. control group) to $+79.4 \%$ ( $\mathrm{p}=\mathrm{NS}$ vs. control group).

\section{Discussion}

Metabolic syndrome is associated with increased risk of arterial and venous thrombosis (13-15). The pathogenesis of prothrombotic state in the metabolic syndrome is complex and incompletely understood. Increased concentration of some coagulation factors such as fibrinogen, factor VII and tissue factor, deficiency of a major activator of fibrinolysis, tissue plasminogen activator, and excess of fibrinolysis inhibitor, plasminogen activator inhibitor-1 (PAI-1) have been reported in patients with overweight/ obesity. In addition, platelet aggregation is augmented in obese animals and humans (16), as evidenced by increased ADP-induced platelet aggregation (6), higher urinary 11-dehydro- $\mathrm{TXB}_{2}$ excretion (8), plasma P-selectin (17) and soluble CD40 ligand levels (18). Platelets of obese subjects are resistant to inhibitory effects of prostacyclin and adenosine (which elevate intracellular cAMP) and nitric oxide (which elevates intracellular cGMP), as well as to these cyclic nucleotides themselves $(19,20)$. Moreover, metabolic syndrome is associated with reduced sensitivity of platelets to at least two groups of antiplatelet drugs, acetylsalicylic acid and $\mathrm{PY}_{12}$ receptor inhibitors, thienopyridines (21). Increased endothelial cell-platelet interaction has been observed in visceral adipose tissue in murine models of obesity (22).

Several studies have demonstrated that leptin augments ADP-, thrombin- or collagen-induced platelet adhesion and/or aggregation in vitro $(5,23,24)$. In addition, exogenous leptin administered to wild-type mice augmented vascular thrombosis induced by vessel wall injury $(25,26)$. In the present study we examined the effect of leptin on TXA formation both in vitro and in vivo. TXA $\mathrm{T}_{2}$ is one of the most common platelet agonists, and the most widely prescribed antiplatelet drug, acetylsalicylic acid, reduces platelet activity by inhibiting its synthesis. Apart from stimulating platelets, $\mathrm{TXA}_{2}$ is involved in atherogenesis by inducing vascular smooth muscle cell migration and proliferation (27) and expression of adhesion molecules in endothelial cells (28). To the best of our knowledge, only one previous study 
addressed the effect of leptin on TXA formation. In that study, Dellas et al (29) have demonstrated that leptin increases $\mathrm{TXB}_{2}$ generation by isolated human platelets and augments the stimulatory effect of ADP. Our results are consistent with these data. Although the minimal effective leptin concentration (100 ng/ $\mathrm{ml}$ ) is above physiological level (except in cases of extreme obesity), leptin was applied only for $10 \mathrm{~min}$. It is possible that more prolonged incubation with leptin would have resulted in platelet stimulation even at lower concentrations of this adipokine. Increased urinary TXA 2 metabolites in animals receiving leptin for 8 days at doses which raised its level to values observed in obesity (Table 1) is consistent with this hypothesis. In addition, leptin concentration in the blood perfusing adipose tissue may be locally much higher than in systemic circulation. Thus, it is likely that stimulatory effect of leptin on platelets is relevant in pathological conditions.

Obesity is associated with resistance not only to central anorectic but also to some peripheral effects of leptin (30), which partially results from downregulation of leptin receptors and/ or postreceptor signaling mechanisms due to chronic hyperleptinemia (31). It is controversial if platelets of obese individuals remain sensitive to leptin or become leptin-resistant. Corsonello et al (32) have demonstrated that stimulatory effect of leptin on platelet aggregation is impaired in overweight and obese individuals. In contrast, Corica et al (33) found that leptin-induced platelet aggregation was impaired only in morbidly obese but not in overweight subjects, and Dellas et al (34) observed normal platelet sensitivity to leptin in morbidly obese patients in comparison to normal-weight controls. Herein we demonstrate that both hyperleptinemia induced in lean rats by administration of exogenous leptin and "endogenous" hyperleptinemia in 1-month obese group are associated with resistance to acute $\mathrm{TXA}_{2}$-stimulating effect of leptin in vitro. Despite this resistance, urinary $\mathrm{TXA}_{2}$ metabolites are elevated in hyperleptinemic rats. These results indicate that leptin may still contribute to platelet hyperactivity when hormone level is markedly elevated, although the degree of platelet stimulation is undoubtedly lower than would have been if platelets remained leptin-sensitive.

Surprisingly, resistance to leptin was not observed in the 3-month obese group, although endogenous leptin level tended to be higher in these animals than in the 1-month obese group. We hypothesize that despite possible downregulation of leptin signaling, leptin-induced $\mathrm{TXA}_{2}$ production is augmented by concomitant insulin resistance in these animals. This hypothesis is supported by the following observations: (i) only 3-month obese rats were hyperinsulinemic (and thus presumably insulin resistant), (ii) platelet sensitivity to leptin was augmented in fructose-fed rats which were markedly insulin resistant but relatively normoleptinemic, (iii) rosiglitazone decreased insulin level and platelets' response to leptin in the 3-month obese and fructose-fed but not in either control or hyperleptinemic groups, and (iv) insulin inhibits platelet function and its effect is impaired in the metabolic syndrome $(12,19,35)$. Nevertheless, other explanations cannot be definitely excluded. For example, hyperlipidemia promotes platelet aggregation (36), and both 3-month obese and fructose-fed animals were hyperlipidemic. In addition, rosiglitazone not only reduced insulin level but also improved lipid profile. Whatever the mechanism, the results suggest that PPAR- $\gamma$ agonists such as rosiglitazone may beneficially modulate platelet function in obesity/metabolic syndrome by reducing their sensitivity to leptin. In addition, variable degree of insulin resistance may determine platelets' sensitivity to leptin in obese subjects and may explain, at least partially, controversial results of previous studies in this field (32-34).

Interestingly, urinary $\mathrm{TXB}_{2}$ excretion was increased in the hyperleptinemic group. Increased intrarenal $\mathrm{TXA}_{2}$ production may contribute to the development of arterial hypertension and nephropathy due to its vasoconstricting, antinatriuretic and profibrogenic effects. Increased urinary $\mathrm{TXB}_{2}$ excretion was observed in many animal models of hypertension such as spontaneously hypertensive rat, Dahl salt-sensitive rat, fructose- or NO synthase inhibitor-induced hypertension as well as in obese Zucker rats which develop severe nephropathy (37-39). Chronic leptin administration induces arterial hypertension (9) and may induce nephropathy (40-41). It remains to be established if excessive intrarenal $\mathrm{TXA}_{2}$ formation contributes to these leptininduced complications.

There are several limitations of the present study. First, we measured only $\mathrm{TXA}_{2}$ formation and did not assess platelet function directly. TXA formation is an established marker of platelet function and allowed us to examine it both in vitro and in vivo in the same experimental model. Second, we examined the effect of leptin alone and did not address its interaction with other platelet agonists. Third, acutely administered leptin increases natriuresis $(42,43)$. It could be suggested that leptin increased urinary 11-dehydro- $\mathrm{TXB}_{2}$ and 2,3-dinor- $\mathrm{TXB}_{2}$ secondarily to increasing sodium excretion. This possibility seems, however, unlikely for the following reasons: (i) in contrast to 11 -dehydro- $\mathrm{TXB}_{2}$ and 2,3-dinor- $\mathrm{TXB}_{2}$, acutely administered leptin had no effect on $\mathrm{TXB}_{2}$ excretion, (ii) 8-day hyperleptinemia is associated with increased excretion of TXA 2 metabolites whereas natriuresis is reduced in this model (9), and (iii) furosemide increased natriuresis while having no effect on urinary $\mathrm{TXA}_{2}$ metabolites, whereas COX inhibitor, indomethacin, prevented leptin-induced increase in TXA $_{2}$ metabolites but had no effect on leptin-induced increase in natriuresis (unpublished observation). 
In conclusion, we have demonstrated that both acutely and chronically administered leptin increases TXA $_{2}$ formation in the rat. Chronic hyperleptinemia impairs platelets' response to acutely administered leptin if insulin sensitivity is normal, however, the stimulatory effect of leptin is intact if insulin resistance is concomitantly observed. PPAR- $\gamma$ agonists may be useful as adjunctive antiplatelet therapy in obesity/metabolic syndrome because they reduce platelets' sensitivity to leptin by ameliorating insulin resistance.

\section{References}

1. Beltowski J. Leptin and atherosclerosis. Atherosclerosis. 2006; 189: 47-60.

2. Koh KK, Park SM, Quon MJ. Leptin and cardiovascular disease: response to therapeutic interventions. Circulation 2008; 117: 3238-3249.

3. Sattar N, Wannamethee G, Sarwar N, Chernova J, Lawlor DA, Kelly A, et al. Leptin and coronary heart disease: prospective study and systematic review. J Am Coll Cardiol 2009; 53: 167-175.

4. Jennings LK. Role of platelets in atherothrombosis. Am J Cardiol 2009; 103(Suppl):4A-10A.

5. Nakata M, Yada T, Soejima N, Maruyama I. Leptin promotes aggregation of human platelets via the long form of its receptor. Diabetes 1999; 48: 426-429.

6. Ozata M, Avcu F, Durmus O, Yilmaz I, Ozdemir IC, Yalcin A. Leptin does not play a major role in platelet aggregation in obesity and leptin deficiency. Obes Res 2001; 9: 627-630.

7. FitzGerald GA, Pedersen AK, Patrono C. Analysis of prostacyclin and thromboxane biosynthesis in cardiovascular disease. Circulation 1983; 67: 1174-1177.

8. Davì G, Guagnano MT, Ciabattoni G, Basili S, Falco A, Marinopiccoli $\mathrm{M}$, et al. Platelet activation in obese women: role of inflammation and oxidant stress. JAMA 2002; 288: 20082014.

9. Bełtowski J, Jamroz-Wiśniewska A, Wójcicka G, Lowicka E, Wojtak A. Renal antioxidant enzymes and glutathione redox status in leptin-induced hypertension. Mol Cell Biochem 2008; 319: 163-174.

10. Bełtowski J, Wójcicka G, Jamroz-Wiśniewska A. Role of nitric oxide and endothelium-derived hyperpolarizing factor (EDHF) in the regulation of blood pressure by leptin in lean and obese rats. Life Sci 2006; 79: 63-71.

11. Michelson AD. Methods for the measurement of platelet function. Am J Cardiol 2009; 103(3 Suppl): 20A-26A.

12. Trovati M, Mularoni EM, Burzacca S, Ponziani MC, Massucco P, Mattiello L, et al. Impaired insulin-induced platelet antiaggregating effect in obesity and in obese NIDDM pa- tients. Diabetes 1995; 44: 1318-1322.

13. Mertens I, Van Gaal LF. Obesity, haemostasis and the fibrinolytic system. Obes Rev 2002; 3: 85-101.

14. Nieuwdorp M, Stroes ES, Meijers JC, Büller H. Hypercoagulability in the metabolic syndrome. Curr Opin Pharmacol 2005; 5: 155-9.

15. Bodary PF. Links between adipose tissue and thrombosis in the mouse. Arterioscler Thromb Vasc Biol 2007; 27: 2284-91.

16. Anfossi G, Russo I, Trovati M. Platelet dysfunction in central obesity. Nutr Metab Cardiovasc Dis 2009; 19: 440-9.

17. De Pergola G, Pannacciulli N, Coviello M, Scarangella A, Di Roma P, Caringella M, et al. sP-selectin plasma levels in obesity: association with insulin resistance and related metabolic and prothrombotic factors. Nutr Metab Cardiovasc Dis 2008; 18: 227-232.

18. Angelico F, Alessandri C, Ferro D, Pignatelli P, Del Ben M, Fiorello S, et al. Enhanced soluble CD40L in patients with the metabolic syndrome: Relationship with in vivo thrombin generation. Diabetologia 2006; 49: 1169-1174.

19. Anfossi G, Russo I, Massucco P, Mattiello L, Doronzo G, De Salve A, et al.. Impaired synthesis and action of antiaggregating cyclic nucleotides in platelets from obese subjects: possible role in platelet hyperactivation in obesity. Eur J Clin Invest 2004; 34: 482-489.

20. Russo I, Del Mese P, Doronzo G, De Salve A, Secchi M, Trovati $\mathrm{M}$, et al. Platelet resistance to the antiaggregatory cyclic nucleotides in central obesity involves reduced phosphorylation of vasodilator-stimulated phosphoprotein. Clin Chem 2007; 53: 1053-1060.

21. Anfossi G, Russo I, Trovati M. Resistance to aspirin and thienopyridines in diabetes mellitus and metabolic syndrome. Curr Vasc Pharmacol 2008; 6: 313-328.

22. Nishimura S, Manabe I, Nagasaki M, Seo K, Yamashita H, Hosoya $\mathrm{Y}$, et al. In vivo imaging in mice reveals local cell dynamics and inflammation in obese adipose tissue. J Clin Invest 2008; 118: 710-721.

23. Corsonello A, Malara A, De Domenico D, Perticone F, Valenti $\mathrm{A}$, Buemi $\mathrm{M}$, et al. Identifying pathways involved in leptin-dependent aggregation of human platelets. Int J Obes Relat Metab Disord 2004; 28: 979-984.

24. Elbatarny HS, Maurice DH. Leptin-mediated activation of human platelets: involvement of a leptin receptor and phosphodiesterase 3A-containing cellular signaling complex. Am J Physiol Endocrinol Metab 2005; 289: E695-702.

25. Konstantinides S, Schäfer K, Koschnick S, Loskutoff DJ. Leptin-dependent platelet aggregation and arterial thrombosis suggests a mechanism for atherothrombotic disease in obesity. J Clin Invest 2001; 108: 1533-1540. 
26. Bodary PF, Gu S, Shen Y, Hasty AH, Buckler JM, Eitzman DT. Recombinant leptin promotes atherosclerosis and thrombosis in apolipoprotein E-deficient mice. Arterioscler Thromb Vasc Biol 2005; 25: e119-122.

27. Morinelli TA, Zhang LM, Newman WH, Meier KE. Thromboxane $\mathrm{A}_{2}$ /prostaglandin $\mathrm{H}_{2}$-stimulated mitogenesis of coronary artery smooth muscle cells involves activation of mitogen-activated protein kinase and S6 kinase. J Biol Chem 1994; 269: 5693-5698.

28. Dogné JM, Hanson J, Pratico D. Thromboxane, prostacyclin and isoprostanes: therapeutic targets in atherogenesis. Trends Pharmacol Sci 2005; 26: 639-644.

29. Dellas C, Schremmer C, Hasenfuss G, Konstantinides SV, Schäfer K. Leptin signalling and leptin-mediated activation of human platelets: importance of JAK2 and the phospholipases C $\gamma 2$ and A2. Thromb Haemost 2007; 98: 1063-1071.

30. Martin SS, Qasim A, Reilly MP. Leptin resistance: a possible interface of inflammation and metabolism in obesityrelated cardiovascular disease. J Am Coll Cardiol 2008; 52: 1201-10.

31. Scarpace PJ, Zhang Y. Leptin resistance: a prediposing factor for diet-induced obesity. Am J Physiol Regul Integr Comp Physiol 2009; 296: R493-500.

32. Corsonello A, Perticone F, Malara A, De Domenico D, Loddo S, Buemi M, Ientile R, et al. Leptin-dependent platelet aggregation in healthy, overweight and obese subjects. Int $J$ Obes Relat Metab Disord 2003; 27: 566-573.

33. Corica F, Corsonello A, Lucchetti M, Mannucci E, Lucchetti $\mathrm{M}$, Bonfiglio C, et al. Relationship between metabolic syndrome and platelet responsiveness to leptin in overweight and obese patients. Int J Obes 2007; 31: 842-849.

34. Dellas C, Schäfer K, Rohm I, Lankeit M, Ellrott T, Faustin $\mathrm{V}$, et al. Absence of leptin resistance in platelets from morbidly obese individuals may contribute to the increased thrombosis risk in obesity. Thromb Haemost 2008; 100: 1123-1129.
35. Westerbacka J, Yki-Järvinen H, Turpeinen A, Rissanen A, Vehkavaara S, Syrjälä M, et al. Inhibition of platelet-collagen interaction: an in vivo action of insulin abolished by insulin resistance in obesity. Arterioscler Thromb Vasc Biol 2002; 22: 167-172.

36. Pedreño J, Hurt-Camejo E, Wiklund O, Badimón L, Masana L. Platelet function in patients with familial hypertriglyceridemia: evidence that platelet reactivity is modulated by apolipoprotein E content of very-low-density lipoprotein particles. Metabolism 2000; 49: 942-949.

37. Gomi T, Ikeda T, Sasaki Y, Kosugi T, Shibuya Y, Sakurai J. Protective effect of thromboxane synthetase inhibitor on hypertensive renal damage in Dahl salt-sensitive rats. Clin Exp Pharmacol Physiol Suppl 1995; 22: S371-3.

38. Sullivan JC, Sasser JM, Pollock DM, Pollock JS. Sexual dimorphism in renal production of prostanoids in spontaneously hypertensive rats. Hypertension 2005; 45: 406-411.

39. Arvola P, Wu X, Kähönen M, Mäkynen H, Riutta A, Mucha I, Solakivi T, Kainulainen H, Pörsti I. Exercise enhances vasorelaxation in experimental obesity associated hypertension. Cardiovasc Res 1999; 43: 992-1002.

40. Wolf G, Hamann A, Han DC, Helmchen U, Thaiss F, Ziyadeh FN, Stahl RA. Leptin stimulates proliferation and TGF- $\beta$ expression in renal glomerular endothelial cells: potential role in glomerulosclerosis. Kidney Int 1999; 56: 860-872.

41. Lee MP, Orlov D, Sweeney G. Leptin induces rat glomerular mesangial cell hypertrophy, but does not regulate hyperplasia or apoptosis. Int J Obes 2005; 29: 1395-1401.

42. Jackson EK, Li P. Human leptin has natriuretic activity in the rat. Am J Physiol 1997; 272: F333-338.

43. Bełtowski J, Wójcicka G, Górny D, Marciniak A. Human leptin administered intraperitoneally stimulates natriuresis and decreases renal medullary $\mathrm{Na}^{+}, \mathrm{K}^{+}$-ATPase activity in the rat - impaired effect in dietary-induced obesity. Med Sci Monit 2002; 8: BR221-229. 